\title{
PERSPECTIVE OPEN Research and development of Zika virus vaccines
}

\author{
Brian E Dawes ${ }^{1,8}$, Claire A Smalley ${ }^{2,8}$, Bethany L Tiner ${ }^{1,8}$, David WC Beasley ${ }^{1,3,4,5}$, Gregg N Milligan ${ }^{1,3,4,6}$, Lisa M Reece ${ }^{3,4}$, \\ Joachim Hombach ${ }^{7}$ and Alan DT Barrett ${ }^{1,2,3,4}$
}

Zika virus (ZIKV) is a member of the family Flaviviridae, genus Flavivirus, and is transmitted by Aedes sp. mosquitoes. There are three genetic lineages of ZIKV: the East African, West African and Asian lineages. Until recently, Zika fever (ZF) has normally been considered a rare, mild febrile disease, but reports since 2012 have shown potentially severe complications associated with ZIKV infection, including microcephaly and Guillain-Barré syndrome. There are no licensed vaccines for ZIKV; however, many vaccine platforms/approaches that have been utilised for other flavivirus vaccines are being applied to ZIKV. Given the current outbreak of ZIKV in the Americas with its associated risks to pregnancy, we summarise what is known about the virus, how knowledge of currently licensed flavivirus vaccines can be applied to ZIKV vaccine development and the assessments of potential challenges for ZIKV vaccine testing and evaluation.

npj Vaccines (2016) 1, 16007; doi:10.1038/npjvaccines.2016.7; published online 28 July 2016

\section{ZIKA VIRUS EMERGENCE, TRANSMISSION AND DISEASE}

Zika virus (ZIKV) was first isolated from a sentinel Rhesus macaque in the Zika forest of Uganda in $1947 .^{1-3}$ The first isolations of ZIKV from humans occurred in Uganda and Nigeria in $1952 .{ }^{4}$ ZIKV is an enveloped, positive-sense, single-stranded RNA virus in the genus Flavivirus, family Flaviviridae, which includes other medically important mosquito-borne viruses such as dengue (DENV), Japanese encephalitis (JEV) and yellow fever (YFV). ${ }^{2,3,5,6}$ The ZIKV genome consists of $5^{\prime}$ and $3^{\prime}$ noncoding regions and a single open reading frame encoding a polyprotein that is co- and posttranslationally processed to generate three structural proteins (capsid (C), precursor of membrane (prM) and envelope (E)), and seven non-structural (NS) proteins (NS1-NS5) in the gene order: 5'-C-prM-E-NS1-NS2A-NS2B-NS3-NS4A-NS4B-NS5-3'. ${ }^{6}$ Serological studies indicate that there is only one serotype of ZIKV, i.e., it is like JEV and YFV but different from DENV, which has four serotypes. ZIKV is most closely related, both phylogenetically and antigenically, to Spondweni virus (SPOV), which is also a human pathogen. ${ }^{3,5,7}$ Three genetic lineages of ZIKV have been identified that separate geographically: the East African, West African and the Asian lineages. ${ }^{2,5-8}$ Until the early 2000s, its geographic distribution was limited to the equatorial belt around Africa and Asia with circulation predominantly in wild primates and Aedes sp. mosquitoes, with rare 'spillover' infections in humans.'

\section{RECENT ZIKV OUTBREAKS}

In April and May 2007, the first identified outbreak of Zika fever (ZF) occurred in the Federated States of Micronesia (Yap Island). 2,10,11 From the 2000 census data, Yap had a population of 7,391 people. Duffy et al. identified 185 cases of suspected ZIKV disease. Of those, 49 (26\%) were confirmed and 59 (32\%) were probable cases. It was determined that about three quarters of
Yap residents $\left(\sim 5,000\right.$ persons $\left.^{12}\right)$ were infected with ZIKV, and more than 900 people had illness attributable to ZIKV infection. ${ }^{13}$ ZIKV epidemic activity was reported in Gabon during a concomitant chikungunya (CHIKV)/DENV outbreak in 2007 that involved $\sim 20,000$ cases. $^{12,14}$ In 2013-2014, a large epidemic occurred in French Polynesia, which infected $\sim 11 \%$ of the population. 2,15,16 This epidemic spread throughout the Pacific to New Caledonia, Vanuatu, the Cook Islands and the Solomon Islands. 2,3,11,17 The virus was then introduced to Easter Island in $2014 .^{18}$ Zanluca et al. ${ }^{19}$ reported the first identification of ZIKV as the causative agent of an outbreak in northeastern Brazil in early 2015. That report represents the first autochthonous transmission of ZIKV in that country. By May 2015, ZIKV was reported by several other Central and South American countries in the Caribbean. ${ }^{11}$ From 1 January 2007 to 23 March 2016, ZIKV transmission was documented in 61 countries and territories (the outbreak is over in four of these countries). Autochthonous ZIKV transmission has been reported in 34 countries and territories of the Americas. ${ }^{20}$

\section{ZIKV ASIAN LINEAGE STRAINS ASSOCIATED WITH RECENT OUTBREAKS}

The 2007 outbreak in the Federated States of Micronesia was due to a ZIKV strain that was genetically related to the Asian lineage, ${ }^{15,32}$ and the strains isolated during the large epidemic in French Polynesia also belonged to the Asian lineage. ${ }^{16}$ However, based on limited numbers of genomic sequences available, these strains were more closely related to strains previously isolated in South East Asia than the strain isolated from the 2007 outbreak. ${ }^{21,22}$ ZIKV strains isolated from Brazil and the Americas, including those isolated from Easter Island, are closely related to the strains isolated during the outbreaks in French Polynesia, within the Asian lineage. ${ }^{2,11,19,23}$ The strains isolated in

\footnotetext{
${ }^{1}$ Department of Microbiology and Immunology, University of Texas Medical Branch, Galveston, TX, USA; ${ }^{2}$ Department of Pathology, University of Texas Medical Branch, Galveston, TX, USA; ${ }^{3}$ Sealy Center for Vaccine Development, University of Texas Medical Branch, Galveston, TX, USA; ${ }^{4}$ World Health Organization Collaborating Center for Vaccine Research, Evaluation and Training on Emerging Infectious Diseases, University of Texas Medical Branch, Galveston, TX, USA; ${ }^{5}$ Institutional Office of Regulated Nonclinical Studies, University

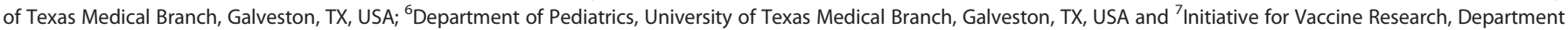
of Immunization, Vaccines and Biologicals, World Health Organization, Geneva, Switzerland.

Correspondence: ADT Barrett (abarrett@utmb.edu)

${ }^{8}$ These authors contributed equally to this work.

Received 6 April 2016; revised 27 May 2016; accepted 9 June 2016
} 
Brazil share more than $99.7 \%$ and $99.9 \%$ of nucleotide and aminoacid identity with the Asian strains, respectively. ${ }^{24}$ Furthermore, there is evidence that ZIKV is continuing to evolve in the Americas. ${ }^{8}$ There are a number of papers examining the evolution of ZIKV in the Americas and the apparent increased virulence of the virus. However, there are probably insufficient genomic sequences with information on passage history, origin and clinical history to make conclusions at this time. Faria et al. ${ }^{11}$ have suggested that ZIKV was introduced into the Americas in the second half of 2013 using a limited number of strains in their analysis, including 23 complete and partial genomic sequences. However, there are over 70 ZIKV genomic sequences available now and a subsequent paper has provided evidence that ZIKV was in Haiti in $2014 .^{25}$ In addition, Zhu et al. reported some genomic changes in their comparison of pre-epidemic African and Asian strains (from mosquito and human isolates). They found a number of amino-acid substitutions throughout the genome and a conformational change in the SL1 structure at the $3^{\prime}$ noncoding region of the epidemic ZIKV strain. There was a possible recombination detected of a NS2B fragment between the Asian lineage of ZIKV and SPOV. ${ }^{26}$ Further investigations are required to elucidate how these changes may be influencing Zika's overall virulence.

\section{ZIKV VECTORS AND TRANSMISSION}

Many Aedes species have had a role in ZIKV transmission and are considered competent vectors including the African species A. africanus, A. apicocoargenteus, A. luteocephalus, A. furcifer, A. vitattus and $A$. aegypti, ${ }^{3,6,27,28}$ In Asia, a sylvatic cycle has not been identified but $A$. aegypti, $A$. hensilli and $A$. albopictus have been shown to be vectors. ${ }^{3}$ In areas with low populations of non-human primates, humans have been suggested as the primary amplification hosts, ${ }^{13}$ with sporadic human ZF cases being reported from the 1960 s to $2007,{ }^{28}$ involving only 14 clinical cases. Furthermore, there is serologic evidence of infection in forest-dwelling birds, horses, cattle, ducks, bats, elephants, goats, hippopotamuses, impala, kongoni, water buffalo, sheep, wildebeest, rodents and zebras. ${ }^{5,21,29-31}$ There are several other potential mechanisms of transmission, i.e., by blood transfusion, sexual transmission or perinatal transmission; ${ }^{5,31-33}$ however, these are believed to be relatively rare when compared with mosquito-borne transmission. Evidence to date indicates a low and transient viraemia in humans, and therefore it is unclear how the virus has managed to spread so widely and quickly in the Americas. Nonetheless, it should be remembered that, at present, there is limited information on the mosquito-vertebrate transmission cycle, particularly in the Americas.

\section{CLINICAL SIGNS AND SYMPTOMS OF ZF COMPARED WITH SPOV, DENV AND CHIKV FEVERS}

As stated above, SPOV is both antigenically and phylogenetically the most closely related virus to ZIKV, and it causes a febrile illness with reported symptoms of a brief fever, headache and malaise. Other reported symptoms include a fine maculopapular rash, conjunctivitis and photophobia. ${ }^{37}$ Similarly, ZIKV infection of humans has generally been considered an acute self-limiting febrile illness characterised by sudden onset of fever, maculopapular rash, arthralgia, retro-orbital pain and conjunctivitis. In some cases, symptoms include myalgia, headache and vomiting. ${ }^{16,17,38,39}$ Clinical presentations of ZF also resemble those that are caused by DENV and the alphavirus CHIKV ${ }^{2,3,16,38}$ and may last up to 10 days (acute phase). ${ }^{13,28}$ However, in contrast to dengue fever (DF), ZF is less severe than DF with headache and malaise being less intense, and signs of haemorrhage and/or shock (as seen for DENV infections) have not been reported. ${ }^{13,16,17}$ Furthermore, conjunctivitis is often present in ZF, but arthralgia is less pronounced. ${ }^{13,16}$ CHIKV presents with high fever, rigors, headache, photophobia, maculopapular rash and severe joint pain; ${ }^{40}$ ZIKV has not been reported to produce rigors or incapacitating joint pain. ${ }^{41}$ Another report described rash presenting 3-5 days after the febrile illness in ZF, but six patients had light asthenia and mild fever 2-3 days before the rash was observed. ${ }^{17}$ The incubation period is thought to be 3-14 days. ${ }^{42}$ Laboratory data include transient leukopaenia and, in some cases, thrombocytopaenia. ${ }^{16,42}$ There are estimations that $80 \%$ of ZIKV infections are asymptomatic, ${ }^{39}$ and severe ZIKV cases involving hospitalisation are uncommon and deaths are rare. ${ }^{41}$

\section{ZIKV AND CONGENITAL ABNORMALITIES}

While ZF has normally been considered to be a mild febrile disease, the full spectrum of pathology is not completely known but has recently expanded to include several neuropathies. Recent reports indicate potentially severe complications associated with ZIKV infection, including microcephaly, central nervous system malformations and Guillain-Barré syndrome (GBS). ${ }^{17,38,43-45}$ Zika congenital syndrome is the term used to refer to severe congenital malformations in humans infected by ZIKV. It describes features of congenital microcephaly: facial disproportionality and cutis girata, in addition to hypertonia or spasticity, hyper-reflexia, irritability, tremors and convulsions. ${ }^{46}$ In addition, severe microcephaly and other brain abnormalities observed in many infants were consistent with ZIKV infection occurring during the first- or early-second trimester of pregnancy. In the 2015 Brazilian outbreak, there were accounts of an increasing number of infants born with microcephaly in ZIKV-affected areas. To obtain data concerning pregnancies (e.g., exposure history, symptoms and laboratory tests), physical examinations of infants, and any additional studies, the Brazilian Society of Medical Genetics established the Zika Embryopathy Task Force. The task force reviews all incident cases of microcephaly as well as infants whose mothers are suspected of having ZIKV infection. The focus of the Task Force is to investigate the possible association of microcephaly with ZIKV infection during pregnancy and establish a standardised registry for incident microcephaly cases. ${ }^{47}$ As of 17 March 2016, a total of 6,671 microcephaly cases in Brazil are suspected to be associated with a ZIKV infection. Investigations have been concluded for 2,212 cases and 863 were confirmed for evidence of ZIKV infection. In addition, Ventura et al. ${ }^{45}$ have reported ophthalmic data from three children in Brazil with microcephaly born after the ZIKV outbreak. Here the infants had macular pigment mottling, foveal reflex loss, macular neuroretinal atrophy (one child) and cerebral calcifications (detected by CT scans) due to presumable intrauterine ZIKV infection. ${ }^{45}$ French Polynesian health authorities reported an increase in central nervous system malformation in fetuses and newborns during the ZIKV outbreaks. In that report, sera from 4 of these 17 pregnant women were positive by IgG for flavivirus, which may suggest asymptomatic or subclinical infections. ${ }^{48}$ ZIKV has been reported in Colombia since October 2015, and there have been over 30,000 cases of reported ZIKV infections where more than 5,000 of these cases were in pregnant women. As of 30 April 2016, there are no reports of associated cases of microcephaly in that country. ${ }^{49}$ In April 2016, because of the available evidence, the CDC concluded that there was sufficiently robust scientific evidence to establish a causal relationship between prenatal ZIKV infection and microcephaly and other serious brain anomalies. ${ }^{50,51}$

\section{ZIKV AND GBS}

GBS is an autoimmune disease characterised by progressive muscle weakness that can result in respiratory failure. ${ }^{52}$ Since October 2015, five countries or territories have reported increased $\mathrm{GBS}^{53}$ associated with ZIKV transmission in those areas. Current 
evidence suggests that the incidence of GBS is 8-10-fold higher in ZIKV IgM-positive individuals compared with the uninfected population. During the outbreak in French Polynesia, 73 cases of GBS and other neurologic conditions were reported among all ages of people. ${ }^{38}$ One study estimated that the rate of GBS after ZIKV infection was $\sim 1$ per 4,000 infections. Patients with Zikaassociated GBS deteriorate rapidly with the average length of intensive care unit stay as 35 days. ${ }^{52}$ There have been unusual increases in reported GBS cases in the Americas, with Brazil reporting 121 cases, Venezuela describing 15 cases, El Salvador having 46 cases and Martinique reporting 2 confirmed cases. ${ }^{38,54}$ A new report from the recent American Academy of Neurology 2016 Annual Meeting describes a potential link between acute disseminated encephalomyelitis (ADEM) and ZIKV. Between December 2014 and June 2015, six patients in Pemambuco, Brazil, presented in the hospital with fever, rash, pruritus, myalgia, arthralgia and conjunctival hyperaemia. Two patients presented with ADEM and four with GBS. All six cases were positive for ZIKV. This is the first report of ADEM associated with the virus. Both ADEM and GBS are immunologic conditions triggered by infections. These reports of ADEM and GBS suggest that ZIKV may be triggering an immunological response causing these neurological conditions. ${ }^{55}$ Such immunological responses might reflect putative immunological cross-reactivity to glycolipids or peptide sequences shared between virus and host, or mechanisms such as epitope spreading, or bystander activation in genetically susceptible hosts. Alternatively, GBS may develop mainly as a consequence of the neurotropism of ZIKV. ${ }^{56-58}$ Nevertheless, links between ZIKV infection and GBS have not yet been proven since the ECDC Rapid Risk Assessment published their report on 10 December $2015 .^{38}$ This is not surprising because GBS can be secondary to other immune events and other virus infections. ${ }^{59} \mathrm{At}$ present, the relationship between ZIKV infection and GBS is incompletely understood, and more studies are needed to firmly establish a mechanism-based link.

\section{ZIKV DIAGNOSTIC PROCEDURES}

The presumptive diagnosis of ZIKV is typically clinical with confirmatory laboratory tests performed using serum, saliva and/ or urine samples. If samples are collected within 1-3 days of fever onset, NS1 antigen may be detected in serum and reverse transcription PCR (RT-PCR) can be used to detect a specific region of the viral genome that includes NS5. ${ }^{43}$ ZIKV RNA can also be detected in both urine and saliva samples collected within the first $3-5$ days of fever onset. ${ }^{22-24}$ Current testing recommendations are to obtain RT-PCR results from urine or saliva within the first 5 or 6 days of illness. ${ }^{5}$ Serological tests, such as enzyme-linked immunosorbent assays and immunofluorescence assays can be performed to detect anti-ZIKV IgM and IgG antibodies. ${ }^{62,63}$ For confirmation of enzyme-linked immunosorbent assay-positive and RT-PCR-negative samples, a plaque reduction neutralisation test ${ }^{5}$ can be performed at reference laboratories. Nevertheless, secondary flavivirus infections potentially complicate plaque reduction neutralisation test-based diagnostics because of the induction of broadly cross-reactive antiflavivirus antibody responses ${ }^{13}$ (e.g., including ZIKV there at least 10 flaviviruses in Brazil). The World Health Organization (WHO) has established an Emergency Use and Assessment Listing for diagnostic tests in response to which 10 tests, both PCR- and serology-based, have been submitted (http://www.who.int/diagnostics_laboratory/eualzika-virus/160520_weekly_update.pdf?ua = 1).

\section{CURRENT ZIKV INFECTION MANAGEMENT}

There are neither antiviral treatments nor vaccines for ZIKV available currently. ${ }^{9,64}$ In addition, there are no standardised reference reagents (antigens or antibodies), although these are in development. Treatment is thus symptomatic such as treating pain and fever with the use of acetaminophen (paracetamol). ${ }^{64}$ Current preventions include personal protection, e.g., mosquito repellent, avoidance of mosquito bites (especially by pregnant women and ZIKV-infected individuals), wearing long pants and/or shirts with long sleeves, screens, bed nets and mosquito surveillance and control methods. ${ }^{5,32,64}$

\section{CURRENT VACCINE DEVELOPMENT EFFORTS}

Although there are no licensed vaccines for $Z I K V,{ }^{9}$ many vaccine platforms/approaches that have been utilised for vaccine research for other flaviviruses are being applied to ZIKV. Table 1 shows platforms/technologies being used in nonclinical development of flavivirus vaccines, ${ }^{65}$ and many groups are investigating the same technologies for a ZIKV vaccine.

\section{PROTECTIVE IMMUNITY AGAINST ZIKV}

The current understanding of protective immune responses to ZIKV is limited, and is derived primarily from human data

Table 1. Examples of potential ZIKV vaccine strategies based on studies with other flaviviruses

Technological approach Antigen ${ }^{\text {a }}$

Recombinant subunit EDIII-p64k fusion proteins and EDIII-capsid vaccines fusion proteins expressed in Escherichia coli Bivalent 80E-STF2 fusion proteins expressed in baculovirus/insect cells

E protein

$80 \mathrm{E}$ protein

EDIII protein expressed in E. coli

DNA vaccines $\mathrm{prM} / \mathrm{E}$ expressed from plasmid vector

VLP vaccines

prM/E

EDIII-HBsAg VLPs or ectoE-based VLPs expressed in Pichia pastoris MVA-VLP

Recombinant chimeric YF 17D backbone live vaccines

DENV-2 backbone JE SA14-14-2 backbone Host range mutations Targeted mutation (2'-O-Methyltransferase mutant)

DENV-4 backbone

EDIII expressed from live-attenuated measles virus vector

Single round replicating viruses

E85 expressed from single-cycle VEE virus vector

RepliVax

Virus-vectored

Live adenovirus 4/7 oral vector

vaccines

Purified inactivated Purified inactivated

virus vaccines

Purified inactivated virus (+Venezuelan equine encephalitis - particle adjuvant)

Abbreviations: DENV, dengue virus; MVA-VLP, modified vaccinia ankaravirus like particle; VEE, Venezuelan equine encephalitis; ZIKV, Zika virus. ${ }^{\mathrm{a}} 80 \mathrm{E}$ and $\mathrm{E} 85$ refer to the N-terminal $80 \%$ and $85 \%$ of the $\mathrm{E}$ protein, respectively, which is the ectodomain of the $E$ protein (also termed ectoE by some). EDIII is domain III of the ectodomain. prM/E is premembrane and envelope protein genes. 
following infection and from comparison with other flaviviruses. Epidemiological evidence suggests that there is a period of time following acute infection in which patients are immune to reinfection, although the long-term durability of this protection is not known. ${ }^{13,15}$ On the basis of evidence from other flavivirus infections, natural infection with ZIKV is likely to result in life-long immunity. Further evidence shows that for secondary (i.e., Zika following some other flavivirus) infections, at least a fourfold rise in neutralising antibody titre against ZIKV occurs between the acute to convalescent phase. ${ }^{66}$ In analysing patient serum from the 2013-2014 ZIKV outbreak in Micronesia, the predominant acute-phase antibody was IgM that was cross-reactive with a variety of flaviviruses, which transitioned to cross-reactive IgM and IgG antibodies in the convalescent phase. ${ }^{62}$ In April 2007, an epidemic ZIKV was noted on Yap Island. Some patients had detectable IgM levels as early as day 3 post onset of symptoms, and some produced neutralising antibody by day 5 after onset of symptoms as measured by neutralisation assays. ${ }^{62}$ Altogether, these data suggest that neutralising antibodies are essential for protection. This would be expected, as available data suggest that for three of the currently licensed flavivirus vaccines (YFV, JEV and tick-borne encephalitis (TBEV)), neutralising antibodies represent a correlate of protection and that a neutralisation titre of 1 in 5 or 1 in 10 is protective. ${ }^{67}$ For dengue, and the recently licensed recombinant live tetravalent vaccine, the situation is more complex and a correlate of protection has not been identified yet. Cytokine profiles taken from acute and convalescent human patient serum are indicative of the development of cell-mediated immunity reflected by increased levels of polyfunctional T helperassociated cytokines and chemokines. ${ }^{16}$ More work is required to define a relationship between cell-mediated immunity and immune protection against ZIKV, including for long-term immunity.

\section{LIVE ATTENUATED FLAVIVIRUS VACCINES}

Live attenuated vaccines (LAVs) offer protective immunity after one or a few doses because of multiplication of the vaccine in the host, which stimulates T and B cells. ${ }^{68}$ Although LAVs are effective, an important potential limitation is safety, as there is always a risk of reversion to virulence. ${ }^{69}$ However, LAVs against flaviviruses, such as JE SA14-14-2 or YF 17D, have had significant success, inducing robust protective immunity after one dose because of the capability of replication within the host, thereby imitating natural infection. ${ }^{65}$ Recently, chimeric recombinant LAVs for JEV and DENV have been licensed based on the YF 17D vaccine virus backbone with the YF 17D prM/E genes replaced by those of JEV and DENV, respectively. ${ }^{70-72}$ There is little doubt that a LAV for ZIKV would be a very effective vaccine; however, our understanding of ZIKV clinical disease is limited, and animal models are still in the discovery phase. Consequently, identifying an attenuated phenotype for a ZIKV LAV will be very demanding and the evaluation process likely complicated.

\section{INACTIVATED FLAVIVIRUS VACCINES}

Inactivated flavivirus vaccines have been licensed for JEV, TBEV ${ }^{8}$ and Kyasanur Forest disease. Inactivated vaccines require multiple doses and very high virus titres/quantity of protein to induce a protective immune response. There is very little information available on the Kyasanur Forest disease vaccine. ${ }^{73,74}$ However, those licensed for JEV and TBEV ${ }^{8}$ require two doses and very high virus titres $\left(10^{8}\right.$ p.f.u. equivalent per dose or $\sim 6 \mu \mathrm{g}$ purified, inactivated JEV proteins and $3 \mu \mathrm{g}$ inactivated TBEV proteins) and are mostly adjuvanted with alum ( $250 \mathrm{mg}$ aluminium hydroxide) to induce a protective immune response for 3-5 years. Clearly, development of inactivated ZIKV vaccines could follow the development path utilised for inactivated JEV and TBEV vaccines with a two-dose regimen to give protective immunity, and duration of immunity followed post licensure. In addition, adjuvants, including oil-in-water emulsions, have the potential for dose-sparing, and preliminary clinical data have been collected for inactivated flavivirus vaccines, including candidate dengue vaccines.

\section{NON-REPLICATING, SUBUNIT FLAVIVIRUS VACCINES}

There are no licensed flavivirus subunit, non-replicating vaccines. However, there are a number of candidates, including recombinant subunit vaccines and DNA vaccines, that are all promising technologies and may be suitable for application to ZIKV, including special populations. ${ }^{65}$ Nevertheless, as mentioned earlier, the non-replicating nature of these types of vaccines requires multiple doses and use of an adjuvant to establish and maintain immunity, which adds complexity to the roll-out of such vaccines.

Recombinant subunit vaccines produce robust immunity to specific antigenic epitopes; however, more work is required to determine which components of ZIKV stimulate neutralising antibody. On the basis of research with other medically important flaviviruses, the envelope (E) protein would be a critical component. Such vaccine development would be aided by the availability of structural data. Indeed, recent studies have reported the cryo-electron microscopic structure of ZIKV mature virions based on virus grown in Vero cells, ${ }^{75,76}$ demonstrating that the overall structure of ZIKV virions is similar to that of DENV. Furthermore, the structures of the $E$ protein complexed with a neutralising monoclonal antibody Fab fragment ${ }^{77}$ and $\mathrm{NS}^{78}$ (protein that induces complement-fixing antibodies) have been solved.

DNA vaccines offer great potential interest for relative ease of development and manufacture, ${ }^{79}$ and have provided a platform that induces robust cellular and humoral immune responses in small animals and non-human primates. ${ }^{52}$ Unfortunately, based on DENV they are yet to show promising results in limited clinical studies. $^{80}$

\section{ANIMAL MODELS FOR ZIKV STUDIES}

There is no established animal model for ZIKV studies, which makes preclinical evaluation of candidate ZIKV vaccines in animals difficult. ZIKV infection results in a neurotropic and fatal disease in suckling and weanling immunocompetent mice following intracerebral inoculation. ${ }^{1}$ Histopathology of mouse brains shows both cellular degeneration and the development of inclusion bodies in the central nervous system. ${ }^{27}$ Immunocompetent animals, including rhesus monkeys, rabbits and guinea pigs, exhibit no clinical disease when infected; however, rhesus monkeys will develop transient viraemia and pyrexia. ${ }^{1}$ Immunocompetent animals develop viraemia and produce antibodies to ZIKV. As it has been possible to develop non-neurotropic disease for $\mathrm{YF}^{81}$ and DEN viruses ${ }^{53-55,82}$ in immunocompromised mice, a number of groups have developed mouse models for ZIKV. Recently, five papers have described lethal ZIKV mouse models utilising mice deficient in either interferon (IFN)- $\alpha / \beta$ receptors $(A 129)^{39,85,86}$ or both IFN- $\alpha / \beta$ and IFN- $\gamma$ receptors (AG129). ${ }^{87,88}$ The $A 129$ mouse model appears to have limitations for vaccine studies as clinical signs are only seen in young mice, whereas ZIKV causes clinical disease in older AG129 mice, which would be more suitable for vaccine immunisation and challenge studies. However, more studies are required before conclusions can be made on potential mouse models. Nonetheless, based on studies with other flaviviruses, there are benefits and disadvantages of utilising immunocompromised mice for vaccine development. ${ }^{83}$ Whereas live vaccine candidates appear to induce a strong adaptive immune response, limited studies show that AG129 mice are not ideal to evaluate 
recombinant protein-based DENV vaccine candidates; ${ }^{83,84}$ however, further work is needed in this area. There are also studies underway to develop non-human primate models of ZIKV infection and in utero transmission. ${ }^{89}$

\section{PREFERRED CHARACTERISTICS OF ZIKV VACCINES FOR USE IN ENDEMIC AREAS AND/OR OUTBREAK RESPONSE}

Predicting the future use of a licensed ZIKV vaccine is difficult in the absence of a better understanding of ZIKV epidemiology and transmission cycles. It is conceivable that ZIKV vaccine(s) could be a part of the routine vaccination schedule in endemic areas. An emergency use target product profile is being developed by WHO to respond to current or future major epidemics.

There are two possibilities of transmission cycles in the Americas, either the cycle is primarily human-mosquito-human (e.g., DENV) or it is zoonotic involving an animal-mosquito cycle with humans being incidental/dead-end hosts (e.g., JEV), and the approach to vaccination would likely differ depending on the transmission cycle. In the emergency context, candidate vaccines would likely be prioritised for women of childbearing age and pregnant women, given the association of ZIKV with microcephaly in neonates. Nonetheless, such vaccine development is very challenging.

Ideally, the vaccine(s) produced should be suitable for use and distribution in low- and middle-income countries, such as Brazil. This requires consideration by developers of issues such as thermostability, need for temperature control in the supply chain and cost of post-licensure clinical studies to evaluate the safety and efficacy of the final vaccine(s) in endemic countries that may lack infrastructure to support those studies. The definition of product characteristics in context of the target product profile for a ZIKV vaccine will aid in focusing development efforts and investment in response to the current outbreak.

\section{CHALLENGES FOR DEVELOPMENT AND TESTING OF ZIKV VACCINE CANDIDATES}

There are more than 60 research institutes and companies working on products to combat the spread of ZIKV. ${ }^{90}$ The pathway to a ZIKV vaccine is still in the nonclinical stage. ${ }^{91}$ To date, there is only one published paper $^{92}$ on ZIKV vaccine candidates, and very little information is available regarding induction of immunity against ZIKV in humans or animals. However, the related flavivirus, DENV, is the topic of intensive vaccine research with a recently licensed product and several vaccine candidates in clinical and preclinical development. ${ }^{65,90}$ Several of these vaccine platforms could potentially be applied to ZIKV vaccine development. Notably, ChimeriVax yellow feverJapanese encephalitis vaccine has been licensed since 2010 (under the trade name IMOJEV) and the ChimeriVax-yellow fever dengue LAV vaccine from Sanofi Pasteur (trade name DENGVAXIA) containing DENV structural genes in a YFV backbone has completed phase III clinical trials and been licensed in several countries. ${ }^{71,93}$ Additional recombinant or chimeric LAV DENV vaccines have entered phase II or III trials, and purified inactivated, recombinant subunit and DNA vaccines have entered phase I trials. ${ }^{93}$ Suitability of these approaches for the target population(s) needs to be considered. Using one of these platforms utilising ZIKV antigens should be technically feasible and would potentially result in faster vaccine development because of the use of regulatory pathways established for these platforms during JEV and DENV vaccine development. Before these platforms enter clinical evaluation, nonclinical evaluation must be conducted, which is hampered by the lack of a validated animal model.

An additional gap in knowledge is the mechanism of protective immunity in ZIKV. Long-lasting protective immunity to natural ZIKV infection has not yet been demonstrated, but likely exists similar to that seen for other flaviviruses. Therefore, neutralising antibodies targeted to the $\mathrm{E}$ protein will likely have a major role in protective immunity, although cell-mediated responses will likely also be necessary. In addition, no standardised assays currently exist to measure ZIKV-neutralising antibodies. Ideally, criteria for standardisation of the key parameters for enzyme-linked immunosorbent assay and neutralisation assays should be established before clinical testing.

Once nonclinical studies have addressed the issues above and led to the production of a vaccine candidate, the implementation of phase II/III clinical trials may be logistically difficult because of the sporadic nature of arboviral outbreaks. In the past, ZIKV outbreaks have not typically remained sustained, and this may limit the ability to assess vaccine efficacy in large-scale field trials. This has been a problem for West Nile virus vaccine development. Depending upon the ongoing burden of endemic disease or occurrence of new large outbreaks where candidate vaccines can be evaluated, the definition of appropriate and measureable clinical end point(s) will also affect the design and feasibility of studies to demonstrate clinical efficacy. On the other hand, given the experience with other monovalent flavivirus vaccines, a licensure based on immunological data could be considered (e.g., induction of a particular titre of neutralising antibodies). Identifying a seroprotective neutralising antibody titre may be difficult. The potential role of antiflavivirus antibodies from previous flavivirus infection or vaccination will need to be addressed as well, as this has been noted to modify vaccine response, ${ }^{91}$ and may complicate assaying of ZIKV type-specific neutralisation titres in vaccines.

Another concern will be the use of ZIKV vaccine to protect pregnant women because of the association of ZIKV infection with fetal neurological defects such as microcephaly. Pregnant women are a special population, and therefore any ZIKV vaccine will need to undergo appropriate nonclinical and clinical safety testing before undergoing trials in pregnant women. An important consideration is the method of immunisation administration to this target population. Later phase clinical trials may investigate maternal immunisation; therefore, protection could be conferred to the pregnant women and their unborn fetuses. Administration of inactivated vaccines during pregnancy would provide passive protection to the baby via transfer of vaccine-induced immunoglobulin across the placenta. ${ }^{68}$ In the short-term and emergency context, the vaccination of pregnant women might be acceptable in the absence of a specific indication and based on a more restricted set of data, requiring a thorough risk benefit assessment, and the absence of a contraindication on the product label. In the longer term, one approach that may be considered is administration of a vaccine that gives long-term protection before childbearing age such that it would protect against congenital disease. Specifically, a LAV viraemia could stimulate protective immunity that is sufficiently robust to prevent any viraemia during subsequent infection, assuming that viraemia is a risk for infection of the fetus even in the absence of clinical disease in the mother.

For all vaccine candidates, the assessment of the risk of triggering GBS will constitute an adverse event of special interest, and will need to be studied throughout the development programme, and monitoring post registration.

Like West Nile virus and CHIKV before it, the emergence of ZIKV since 2007 and the explosive outbreak currently occurring in the Americas highlight the potential for relatively obscure arboviruses to rapidly and unexpectedly expand both their geographic range and their importance as agents of human disease. Whether this emergence and the occurrence of apparently severe diseases are because of virus, host or other factors remains to be determined. The global response to ZIKV will presumably leverage lessons learned from the response to the 2014-2015 Ebola virus outbreak in West Africa to facilitate rapid development and testing of new vaccine candidates and other products for prevention/treatment 
of infection or severe disease. Although many questions remain to be answered regarding the specifics of ZIKV infection and disease, the development and testing of ZIKV vaccines will benefit from recent experience with other flavivirus vaccine candidates that have utilised a wide range of platforms and provided insights into the induction of protective, long-lasting immunity to flaviviruses.

\section{COMPETING INTERESTS}

The authors declare no conflict of interest.

\section{REFERENCES}

1. Dick, G. W. A., Kitchen, S. F. \& Haddow, A. J. Zika Virus (I). Isolations and serological specificity. Trans. R Soc. Trop. Med. Hyg. 46, 509-520 (1952).

2. Tognarelli, J. et al. A report on the outbreak of Zika virus on Easter Island, South Pacific, 2014. Arch. Virol. 161, 665-668 (2015).

3. Wong, P.-S. J., Li, M. I., Chong, C.-S., Ng, L.-C. \& Tan, C.-H. Aedes (Stegomyia) albopictus (Skuse): a potential vector of Zika virus in Singapore. PLoS Negl. Trop. Dis. 7, e2348 (2013).

4. Dick, G. W. Zika virus (II). Pathogenicity and physical properties. Trans. R. Soc. Trop. Med. Hyg. 46, 521-534 (1952).

5. Kelser, E. A. Meet dengue's cousin, Zika. Microbes Infect. 18, 163-166 (2015).

6. Faye, O. et al. Molecular evolution of Zika virus during its emergence in the 20th century. PLoS Negl. Trop. Dis. 8, e2636 (2014).

7. Haddow, A. D. et al. Genetic characterization of Zika virus strains: geographic expansion of the Asian lineage. PLoS Negl. Trop. Dis. 6, e1477 (2012).

8. Lanciotti, R. S., Lambert, A. J., Holodniy, M., Saavedra, S. \& del Carmen Castillo Signor, L. Phylogeny of Zika Virus in Western Hemisphere, 2015. Emerg. Infect. Dis. 22, 933-935 (2016).

9. Fauci, A. S. \& Morens, D. M. Zika virus in the Americas-yet another arbovirus threat. N. Engl. J. Med. 374, 601-604 (2016).

10. Craig, A. T., Butler, M. T., Pastmore, R., Paterson, B. J. \& Durrheim, D. N. Update on Zika virus transmission in the Pacific islands, 2007 to February 2016 and failure of acute flaccid paralysis surveillance to signal Zika emergence in this setting. Bull. World Health Organ; epub ahead of print 19 February 2016, doi: http://dx.doi.org/ 10.2471/BLT.16.1711892 (2016).

11. Faria, N. R. et al. Zika virus in the Americas: early epidemiological and genetic findings. Science 352, 345-349 (2016).

12. Grard, G. et al. Zika Virus in Gabon (Central Africa)-2007: a new threat from Aedes albopictus? PLoS Negl. Trop. Dis. 8, e2681 (2014).

13. Duffy, M. R. et al. Zika virus outbreak on Yap Island, Federated States of Micronesia. N. Engl. J. Med. 360, 2536-2543 (2009).

14. Leroy, E. M. et al. Concurrent chikungunya and dengue virus infections during simultaneous outbreaks, Gabon, 2007. Emerg. Infect. Dis. 15, 591-593 (2009).

15. Cao-Lormeau, V.-M. et al. Zika virus, French Polynesia, South Pacific, 2013. Emerg. Infect. Dis. 20, 1084-1086 (2014).

16. Tappe, D. et al. Cytokine kinetics of Zika virus-infected patients from acute to reconvalescent phase. Med. Microbiol. Immunol. (Berl.) 205, 269-273 (2015).

17. Gourinat, A.-C., O'Connor, O., Calvez, E., Goarant, C. \& Dupont-Rouzeyrol, M. Detection of Zika virus in Urine. Emerg. Infect. Dis. 21, 84-86 (2015).

18. Schwan, K. Zika Virus-Pacific (07): chile (Easter Island), French Polynesia (International Society for Infectious Diseases, 2014).

19. Zanluca, C. et al. First report of autochthonous transmission of Zika virus in Brazil. Mem. Inst. Oswaldo Cruz 110, 569-572 (2015).

20. World Health Organization. Zika Virus, Microcephaly and Guillain-Barré Syndrome. Situation Report 1-13 (2016). http://apps.who.int/iris/bitstream/10665/204690/1/ zikasitrep_24Mar2016_eng.pdf?ua=1 (28 April 2016).

21. Buathong, R. et al. Detection of Zika virus infection in Thailand, 2012-2014. Am. J. Trop. Med. Hyg. 93, 380-383 (2015).

22. Alera, M. T. et al. Zika virus infection, Philippines, 2012. Emerg. Infect. Dis. 21, 722-724 (2015).

23. Calvet, G. A. et al. First detection of autochthonous Zika virus transmission in a HIV-infected patient in Rio de Janeiro, Brazil. J. Clin. Virol. 74, 1-3 (2016).

24. Enfissi, A., Codrington, J., Roosblad, J., Kazanji, M. \& Rousset, D. Zika virus genome from the Americas. Lancet 387, 227-228 (2016).

25. Lednicky, J. et al. Zika virus outbreak in Haiti in 2014: molecular and clinical data. PLoS Negl. Trop. Dis. 10, e0004687 (2016)

26. Zhu, Z. et al. Comparative genomic analysis of pre-epidemic and epidemic Zika virus strains for virological factors potentially associated with the rapidly expanding epidemic. Emerg. Microbes Infect. 5, e22 (2016).

27. Weinbren, M. \& Williams, M. Zika virus: further isolations in the zika area, and some studies on the strains isolated. Trans. R. Soc. Trop. Med. Hyg. 52, 263-268 (1958).
28. Musso, D. et al. Potential for Zika virus transmission through blood transfusion demonstrated during an outbreak in French Polynesia, November 2013 to February 2014. Eurosurveillance 19, 20761 (2014).

29. Darwish, M. A., Hoogstraal, H., Roberts, T. J., Ahmed, I. P. \& Omar, F. A seroepidemiological survey for certain arboviruses (Togaviridae) in Pakistan. Trans. $R$. Soc. Trop. Med. Hyg. 77, 442-445 (1983).

30. Henderson, B. E., Hewitt, L. E. \& Lule, M. Serology of Wild Mammals 48-51 (East African Printer, 1968).

31. Malone, R. W. et al. Zika virus: medical countermeasure development challenges. PLoS Negl. Trop. Dis. 10, e0004530 (2016).

32. Higgs, S. Zika virus: emergence and emergency. Vector-Borne Zoonotic Dis. 16, 75-76 (2016).

33. Besnard, M., Teissier, S., Cao-Lormeau, V. \& Musso, D. Evidence of perinatal transmission of Zika virus, French Polynesia, December 2013 and February 2014. Euro Surveill. 19, 1-4 (2014).

34. Ayres, C. F. J. Identification of Zika virus vectors and implications for control. Lancet Infect. Dis. 16, 278-279 (2016).

35. Deckard, D. T. et al. Male-to-male sexual transmission of Zika virus-Texas. January 2016. MMWR Morb. Mortal. Wkly. Rep. 65, 372-374 (2016).

36. McCarthy, M. Zika cases climb to 147 in US and Puerto Rico. BMJ 352, i1212 (2016).

37. Wolfe, M. S., Calisher, C. H. \& McGuire, K. Spondweni virus infection in a foreign resident of Upper Volta. Lancet 320, 1306-1308 (1982).

38. European Centre for Disease Prevention and Control (ECDC). Zika Virus Disease Epidemic: Potential Association with Microcephaly and Guillain-Barre Syndrome. First Update, 21 January 2016 (ECDC, Stockholm, 2016).

39. Rossi, S. L. et al. Characterization of a novel murine model to study Zika virus. Am. J. Trop. Med. Hyg. 94, 1362-1369 (2016).

40. Schwartz, O. \& Albert, M. L. Biology and pathogenesis of chikungunya virus. Nat. Rev. Microbiol. 8, 491-500 (2010).

41. Paixão, E. S. et al. History, epidemiology, and clinical manifestations of zika: a systematic review. Am. J. Public Health 106, 606-612 (2016).

42. Focosi, D., Maggi, F. \& Pistello, M. Zika virus implications for public health. Clin. Infect. Dis. ciw210, 1-7 (2016).

43. Martines, R. B. Evidence of Zika virus infection in brain and placental tissues form two congenitally infected newborns and two fetal losses-Brazil, 2015. MMWR 65 159-160 (2016).

44. Heymann, D. L. et al. Zika virus and microcephaly: why is this situation a PHEIC? Lancet 387, 719-721 (2016).

45. Ventura, C. V., Maia, M., Bravo-Filho, V., Góis, A. L. \& Belfort, R. Zika virus in Brazil and macular atrophy in a child with microcephaly. Lancet 387, 228 (2016).

46. Miranda-Filho, D. et al. Initial description of the presumed congenital Zika syndrome. Am. J. Public Health 106, 598-600 (2016).

47. Schuler-Faccini, L. et al. Possible association between Zika virus infection and microcephaly_Brazil, 2015. MMWR Morb. Mortal. Wkly. Rep. 65, 59-62 (2016).

48. Cauchemez, S. et al. Association between Zika virus and microcephaly in French Polynesia, 2013-15: a retrospective study. Lancet 387, 2125-2132 (2016).

49. Basarab, M., Bowman, C., Aarons, E. J. \& Cropley, I. Zika virus. BMJ 352, i1049 (2016).

50. Centers for Disease Control and Prevention (CDC). CDC concludes zika causes microcephaly and other birth defects. Media Statement, Wednesday, 13 April 2016. http://www.cdc.gov/media/releases/2016/s0413-zika-microcephaly.html (16 April 2016).

51. Rasmussen, S. A., Jamieson, D. J., Honein, M. A. \& Petersen, L. R. Zika virus and birth defects-reviewing the evidence for causality. N. Engl. J. Med. 374, 1981-1987 (2016).

52. Malkki, H. CNS infections: Zika virus infection could trigger Guillain-Barré syndrome. Nat. Rev. Neurol. 12, 187-187 (2016).

53. European Centre for Disease Prevention and Control (ECDC). Zika Virus Disease Epidemic: Potential Association with Microcephaly and Guillain-Barre Syndrome. Fourth update, 9 March 2016 (ECDC, Stockholm, 2016).

54. Rozé, B. et al. Zika virus detection in urine from patients with Guillain-Barré synbdrome on Martinique, January 2016. Euro Surveill 21 (2016). doi: http://dx. doi.org/10.28707/1560-7917.ES.2016.21.9.30154.

55. Hughes, S. Zika Virus Now Linkeed to Autoimmune Neurologic Conditions (Medscape, 2016).

56. Lucchese, G. \& Kanduc, D. Zika virus and autoimmunity: from microcephaly to Guillain-Barré syndrome, and beyond. Autoimmun. Rev. 15, 801-808 (2016).

57. Anaya, J.-M. et al. Zika virus and neurologic autoimmunity: the putative role of gangliosides. BMC Med. 14, 49 (2016).

58. Ricketson, R. \& Weiler, L. J. Zika virus induced neurotropic brain injury: lessons for the study of disease etiology and vaccine development against pathogens. WebmedCentral Virol. 7, WMC005082 (2016). 
59. Araujo, L. M., Ferreira, M. L. B. \& Nascimento, O. J. Guillain-Barré syndrome associated with the Zika virus outbreak in Brazil. Arq. Neuropsiquiatr. 74 253-255 (2016).

60. Musso, D. et al. Detection of Zika virus in saliva. J. Clin. Virol. 68, 53-55 (2015).

61. Fonseca, K. et al. First case of Zika virus infection in a returning Canadian traveler. Am. J. Trop. Med. Hyg. 91, 1035-1038 (2014).

62. Lanciotti, R. S. et al. Genetic and serologic properties of Zika virus associated with an epidemic, Yap State, Micronesia, 2007. Emerg. Infect. Dis. 14, 1232-1239 (2008).

63. Centers for Disease Control and Prevention, Division of Vector-Borne Diseases. Memorandum: Updated diagnostic testing for Zika, chikungunya, and dengue viruses in US Public Health Laboratories (2016). http://www.cdc.gov/zika/pdfs/ denvechikzvikv-testing-algorithm.pdf (20 April 2016).

64. Saxena, S. K., Elahi, A., Gadugu, S. \& Prasad, A. K. Zika virus outbreak: an overview of the experimental therapeutics and treatment. VirusDis. 27, 111-115 (2016).

65. Vannice, K. S., Roehrig, J. T. \& Hombach, J. Next generation dengue vaccines: a review of the preclinical development pipeline. Vaccine 33, 7091-7099 (2015).

66. Olson, J. G., Ksiazek, T. G., Suhandiman \& Triwibowo. Zika virus, a cause of fever in Central Java, Indonesia. Trans. R. Soc. Trop. Med. Hyg. 75, 389-393 (1981).

67. Plotkin, S. A. Correlates of protection induced by vaccination. Clin. Vaccine Immunol. 17, 1055-1065 (2010)

68. Barrett, A. D. T. in Vaccinology: An Essential Guide (eds Milligan, G. \& Barrett, A. D. T.) 175-176 (John Wiley and Sons, Inc, 2015).

69. Ulmer, J. B., Valley, U. \& Rappuoli, R. Vaccine manufacturing: challenges and solutions. Nat. Biotechnol. 24, 1377-1383 (2006).

70. Guy, B., Saville, M. \& Lang, J. Development of sanofi pasteur tetravalent dengue vaccine. Hum. Vaccine 6, 696-705 (2010)

71. Halstead, S. B. \& Thomas, S. J. New Japanese encephalitis vaccines: alternatives to production in mouse brain. Expert Rev. Vaccines 10, 355-364 (2011).

72. Chokephaibulkit, K. et al. Safety and immunogenicity of a single administration of live-attenuated Japanese encephalitis vaccine in previously primed 2- to 5-yearolds and naive 12- to 24-month-olds: multicenter randomized controlled trial. Pediatr. Infect. Dis. J. 29, 1111-1117 (2010).

73. Kasabi, G. S. et al. Coverage and effectiveness of Kyasanur forest disease (KFD) vaccine in Karnataka, South India, 2005-10. PLoS Negl. Trop. Dis. 7, e2025 (2013)

74. Holbrook, M. R. Kyasanur forest disease. Antiviral Res. 96, 353-362 (2012).

75. Kostyuchenko, V. A. et al. Structure of the thermally stable Zika virus. Nature $\mathbf{5 3 3}$ 425-428 (2016).

76. Sirohi, D. et al. The $3.8 \AA$ resolution cryo-EM structure of Zika virus. Science $\mathbf{3 5 2}$ 467-470 (2016).

77. Dai, L. et al. Structures of the Zika virus envelope protein and its complex with a flavivirus broadly protective antibody. Cell Host Microbe 19, 696-704 (2016).

78. Song, H., Qi, J., Haywood, J., Shi, Y. \& Gao, G. F. Zika virus NS1 structure reveals diversity of electrostatic surfaces among flaviviruses. Nat. Struct. Mol. Biol. 23, 456-458 (2016)

79. Yauch, L. E. \& Shresta, S. in Advances in Virus Research Vol. 88, 315-372 (Elsevier, 2014).
80. Porter, K. R. \& Raviprakash, K. Nucleic acid (DNA) immunization as a platform for dengue vaccine development. Vaccine 33, 7135-7140 (2015).

81. Meier, K. C., Gardner, C. L., Khoretonenko, M. V., Klimstra, W. B. \& Ryman, K. D. A mouse model for studying viscerotropic disease caused by yellow fever virus infection. PLoS Pathog. 5, e1000614 (2009).

82. Shresta, S., Sharar, K. L., Prigozhin, D. M., Beatty, P. R. \& Harris, E. Murine model for dengue virus-induced lethal disease with increased vascular permeability. J. Virol. 80, 10208-10217 (2006).

83. Sarathy, V. V., Milligan, G. N., Bourne, N. \& Barrett, A. D. T. Mouse models of dengue virus infection for vaccine testing. Vaccine 33, 7051-7060 (2015).

84. Milligan, G. N. et al. A dengue virus type 4 model of disseminated lethal infection in AG129 mice. PLoS ONE 10, e0125476 (2015).

85. Dowall, S. D. et al. A susceptible mouse model for Zika virus infection. PLoS Negl. Trop. Dis. 10, e0004658 (2016).

86. Lazear, H. M. et al. A mouse model of Zika virus pathogenesis. Cell Host Microbe 19, 720-730 (2016).

87. Zmurko, J. et al. The viral polymerase inhibitor 7-deaza-2'-C-methyladenosine is a potent inhibitor of in vitro Zika virus replication and delays disease progression in a robust mouse infection model. PLoS Negl. Trop. Dis. 10, e0004695 (2016).

88. Aliota, M. T. et al. Characterization of lethal Zika virus infection in AG129 mice PLoS Negl. Trop. Dis. 10, e0004682 (2016).

89. Dudley, D. M. et al. A rhesus macaque model of Asian-lineage Zika virus infection. Nat Commun. 7, 12204 (2016).

90. Cumming-Bruce, N. Zika vaccine still years away, W.H.O. says. The New York Times 9 March 2016. www.nytimes.com/2016/03/10/world/americas/zika-vaccine-still-years-away-who-says.html?_r=0 (4 May 2016).

91. Maurice, J. WHO reveals its shopping list for weapons against Zika. Lancet 387, 733 (2016)

92. Larocca, R. A. et al. Vaccine protection against Zika virus from Brazil. Nature doi:10.1038/nature18952.

93. Schwartz, L. M., Halloran, M. E., Durbin, A. P. \& Longini, I. M. The dengue vaccine pipeline: implications for the future of denque control. Vaccine $\mathbf{3 3}$ 3293-3298 (2015).

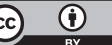

This work is licensed under a Creative Commons Attribution 4.0 International License. The images or other third party material in this article are included in the article's Creative Commons license, unless indicated otherwise in the credit line; if the material is not included under the Creative Commons license, users will need to obtain permission from the license holder to reproduce the material. To view a copy of this license, visit http://creativecommons.org/licenses/ by/4.0/

(c) The Author(s) 2016 